Referencia para citar este artículo: Narodowski, M. \& Snaider, C. (2017). ¿Bebés en las escuelas? Infancias hiperescolarizadas en una cultura prefigurativa. Revista Latinoamericana de Ciencias Sociales, Niñez y Juventud, 15(1), pp. 45-57.

\title{
¿Bebés en las escuelas? Infancias hiperescolarizadas en una cultura prefigurativa*
}

\author{
MARIANO NARODOWSKI ** \\ Profesor-investigador Universidad Torcuato Di Tella, Argentina. \\ CAROLINA SNAIDER *** \\ Teachers College, Columbia University, USA.
}

Artículo recibido en mayo 31 de 2016; artículo aceptado en julio 5 de 2016 (Eds.)

- Resumen (descriptivo): Desde hace más de una década, se observa en la Ciudad de Buenos Aires un notorio crecimiento de la población de niños y niñas de edades entre 0 a 3 años, con escolarización. En este estudio aportamos evidencia estadística corroborando un relevante incremento de las vacantes disponibles, la privatización de la matrícula y la persistencia de una elevada demanda insatisfecha.

Proponemos un andamiaje conceptual para contribuir a responder una pregunta central: ¿cuáles son los cambios culturales y demográficos que están implícitos en el reclamo por más bebés con escolarización? Centramos el estudio en este proceso como emergente de un cambio de época, con nuevas concepciones de infancia y adultez. Por medio del concepto cultura prefigurativa, de Margaret Mead, arriesgamos algunas hipótesis sobre el deterioro del valor del sacrificio adultopaterno.

Palabras clave: Población en edad escolar, educación de la primera infancia, educación privada, Argentina (Tesauro de Ciencias Sociales de la Unesco).

Palabras clave autores: Cultura prefigurativa.

\section{Babies at school? Hyper-schooling of infancy in a prefigurative culture}

- Abstract (descriptive): In the City of Buenos Aires, there has been important growth in preschool enrollment for over a decade. The empirical data evidences a significant increase in enrollment, the privatization of preschool education and the prevalence of unsatisfied demand: What cultural and demographic changes underlie the general demand that more babies should go to school? The emergence of a new era starting with a change in thinking, and a new conceptualization of childhood and adulthood is proposed. Through Margaret Mead's concept of "prefigurative culture", we hypothesize the devaluation of the adult-parental sacrifice.

Key words: School age population, early childhood education, private school, Argentina (Unesco Social Sciences Thesaurus).

Author Key words: Prefigurative culture.

\footnotetext{
Este artículo de reflexión tiene como antecedentes principales dos trabajos: Los desarrollos sobre los cambios en las relaciones sociales posmodernas del autor Mariano Narodowski, contenidos en el libro Un mundo sin adultos, publicado por editorial Debate a principios del 2016; y la investigación "El Nivel Inicial en la Ciudad de Buenos Aires. Lógicas que ordenan ofertas crecientes y diversas" de Carolina Snaider, dirigida por Mariano Narodowski, en la Universidad Torcuatto Di Tella, en 2014. Fechas de inicio y finalización de la investigación que dio origen al artículo: Marzo de 2015 a Febrero de 2016. Número de código, acta o contrato por parte de la entidad que avaló la investigación, no aplica para la Universidad Torcuato Di Tella. Área: Ciencias de la Educación. Subárea: Educación General.

** Doctor en Educación (Universidade Estadual de Campinas). Correo electrónico: mnarodowski@utdt.edu

***_Doctoranda en Educación (Teachers College, Columbia University). Correo electrónico: cs3495@tc.columbia.edu
} 


\section{Bebês nas escolas? Infâncias "hiperescolarizadas" numa cultura pré-figurativa}

- Resumo (descritivo): Nos últimos dez anos, o aumento de crianças escolarizadas em seus primeiros anos de vida cresceu muito na cidade de Buenos Aires. Este estudo traz estatísticas que provam o aumento do número de vagas, a privatização da matrícula e a persistente demanda insatisfeita. Com esses dados, a proposta é desvendar o conceito que contribui a responder uma questão central: Quais são as mudanças culturais e demográficas que ficam por trás das discussões que reclamam por mais e mais bebés na escola? Propõe-se a emergência de uma nova época, com concepções diferentes da infância e da vida adulta. Através do conceito de "cultura pré-figurativa" de Margaret Mead, os autores arriscam a hipótese da deterioração do valor do sacrificio adultopaterno o que torna o ingresso na educação infantil cada vez mais cedo.

Palavras-chave: População em idade escolar, educação infantil, escola particular, Argentina (Tesauro de Ciências Sociais da Unesco).

Palavras-chave autores: Cultura pré-figurativa.

-1. El jardín es escuela; sus alumnos y alumnas son bebés. -2. El Nivel Inicial: Siempre creciente, siempre insuficiente. -3 . Sentidos explícitos: Al jardín se va a aprender -4 . Sentidos implícitos: Adiós al sacrificio paterno. -Lista de referencias.

\section{El jardín es escuela, sus alumnos y alumnas son bebés}

El nivel inicial, primer nivel del sistema educativo en la Argentina, está organizado en dos ciclos: el Jardín Maternal, que abarca las edades de 0 a 2 años, y el Jardín de Infantes, que atiende a los niños y las niñas de 3 a 5 años de edad, siendo la sala de cuatro el primer año de escolaridad elemental obligatoria (Ley de Educación Nacional 26.206, Artículo 18. Congreso Nacional de Argentina, 2006).

Pero esto no siempre fue así. Dicha estructura organizativa se definió a fines de 2014. La Ley 27.045 modificó los artículos 18 y 19 de la Ley de Educación Nacional vigente desde el 2006; de esta manera se adelantó la obligatoriedad escolar desde la sala de cinco años hacia la sala de cuatro, y la meta de universalización quedó estipulada para la sala de tres.

En la legislación previa -Ley Federal de Educación 24.195 vigente desde año 1993-el reconocimiento de los servicios para niños y niñas menores de 3 años, como parte del sistema educativo, estaba desdibujado, y la función del Estado al respecto era ambigua (Mayol-Lasalle, 2002, Ponce, 2006) ${ }^{1}$.

1 En el Artículo 10 de la ley se leía: "Educación Inicial, constituida por el jardín de infantes para niños/as de 3 a 5 años de edad, siendo obligatorio el último año. Las provincias y la Municipalidad de
Los primeros espacios para los niños y las niñas menores de 3 años se reconocieron principalmente como guarderías: no se concibieron como una unidad pedagógica sino como espacios donde se guardaba el cuerpo infantil en tanto sus padres y madres trabajaban, con una fuerte impronta de los cuidados del tipo médico-sanitarios.

$\mathrm{Su}$ expansión comenzó hacia fines de la década del sesenta, cuando la mujer comenzó a ejercer otro rol en la vida pública, y se fueron desarrollando principalmente en el ámbito privado, en las fábricas u otros espacios de trabajo con gran presencia femenina. En lo que respecta a la Ciudad de Buenos Aires (en adelante Caba), aproximadamente durante 30 años -no sin algunos intentos regulatorios fallidos ${ }^{2}-$ las guarderías privadas funcionaron sin ningún tipo de control, excepto fiscal (Argentina, Ministerio

la Ciudad de Buenos Aires establecerán, cuando sea necesario, servicios de jardín maternal para niños/as menores de 3 años".

2 Hasta el año 2001, las guarderías se rigieron por la Ordenanza $\mathrm{N}^{\circ} 25.579$, "Reglamento para habilitación y funcionamiento de guarderías infantiles", vigente desde 1971.

En 1973 la Ley 20.582 "Creación del Instituto de Jardines Maternales Zonales", fue un primer intento en el pasaje de la "guardería" al Jardín Maternal; pero nunca fue reglamentada (Ponce, 2006). También en 1997 se sancionó la Ordenanza N ${ }^{\circ}$ 52.137, a fin de regular la situación de las guarderías e incluirlas al sistema educativo; tampoco fue puesta en práctica. Finalmente, en el año 2001 se sancionó la Ley No 621: "Ley de Regulación de habilitación, funcionamiento y supervisión de instituciones privadas de carácter educativo asistencial, destinadas a niños de 45 días a 4 años, no incorporadas a la enseñanza oficial". 
de Educación y Justicia \& OEA, 1986, Burgos \& Silva, 2013, Brailovsky, Herrera, Salinas \& Santa Cruz, 2002, Diker, 2001, Pitluk, 2002).

En este sentido, la identidad del Nivel Inicial está atravesada desde sus cimientos por una tensión entre su carácter asistencial-pedagógico $\mathrm{y}$, entendiendo que los sentidos no están separados de su enunciación, puede observarse cómo en las diferentes denominaciones con las que se ha ido identificando, se reflejan dichas tensiones fundacionales: asistir-educar; parecerse-diferenciarse de la escolaridad primaria.

En el pasaje del polo asistencial al pedagógico, a partir de los años ochenta del siglo XX, la guardería pasó a identificarse como Jardín Maternal, y la vieja denominación, para los ahora docentes profesionales $\mathrm{y}$ especializados a cargo de los niños y las niñas, ha devenido no solo inadecuada sino hasta ofensiva (Brailovsky, 2006, Caputo \& Gamallo, 2010, Pitluk, 2002).

Actualmente, las instituciones para los más pequeños se denominan Jardín Maternal, Jardín de Infantes o Escuela Infantil -para las instituciones que abarcan ambos ciclos-. Nótese cómo en la misma enunciación se deja asentado algo que años atrás era puesto en cuestionamiento, y que en la batalla por su reconocimiento generó contrapesos introduciendo ordenamientos ${ }^{3}$ propios de niveles educativos superiores: el jardín es escuela.

De todas formas, los usos del lenguaje con que se hace referencia a la estructura actual continúan reflejando la complejidad y parafraseando a Daniel Brailovsky (2006)- el cuestionamiento a los lugares escolares que acarrea el fenómeno de la escolarización a tan temprana edad. En este sentido, señalamos que a diferencia del resto de los niveles del sistema educativo donde cada grupo de alumnos y alumnas se identifica respondiendo al criterio de gradualidad con que se organiza el sistema -por ejemplo, en las escuelas primarias primer

3 Contenidos curriculares de tradición académica, agrupamiento de niños y niñas según sus edades cronológicas, son ordenamientos de los que no ha quedado exento el Nivel Inicial y que reflejan la tendencia a la mimetización con los formatos escolares en la defensa de su carácter educativo (Brailovsky, 2006, Terigi, 2012). grado, segundo grado, etc.- en el ciclo maternal, en lugar de hacer referencia a una característica propia del dispositivo escolar se recurre a características evolutivas de los alumnos y alumnas. Se utilizan categorías basadas en el hecho de que se alimentan de leche materna o que han adquirido habilidades motoras que posibilitan el desplazamiento autónomo: "sala lactantes" para los bebés de 45 días a 1 año de edad y "sala deambuladores" para el grupo de niños y niñas de 1 a 2 años de edad.

Este breve pasaje por los usos del leguaje habilita una aclaración necesaria cuando el objeto de estudio es la escolarización de la primera infancia; tal el caso del presente estudio: el jardín de infantes es escuela y muchos de sus alumnos y alumnas son bebés.

\section{El nivel Inicial: siempre creciente, siempre insuficiente}

En consonancia con el reconocimiento de los jardines maternales y de infantes en tanto instituciones escolares, se observa un notorio crecimiento de la población que asiste a éstas, a cada vez más temprana edad.

Sibien en el presente trabajo tomamos como contexto de análisis el caso de la Caba, cabe mencionar que la escolarización de la primera infancia ha pasado a ser fuente de preocupación internacional y ha adquirido un lugar central en las agendas políticas y educativas de diversos Estados. Tan solo a modo de ilustración, vemos que en 2013 la Comisión Europea recomendó a los Estados miembros de la Unión Europea (UE) invertir en la infancia como modo de "romper ciclos de desventajas" (Unión Europea, 2013, p. 5). Por el lado de la región iberoamericana, el documento Metas 2021 - La educación que queremos para la generación de los bicentenarios, estableció el objetivo de "aumentar la oferta de educación inicial y potenciar su carácter educativo" (Meta general tercera). De esta manera, los países de la región se comprometieron a que el $100 \%$ de los niños y las niñas a partir de los 3 años de edad, y entre el $20 \%$ y el $50 \%$ de los niños y las niñas de 0 a 3 años, reciban atención educativa temprana para el año 2021 (OEI, Cepal \& Secretaría General Iberoamericana, 2010). 
Específicamente en Argentina, aunque la legislación nacional estipula la obligación de la sala de 4 años de edad y propone la universalización de la de 3 (Ley de Educación Nacional 26.206, Artículo 18 y 19. Congreso Nacional de Argentina, 2006), en la Caba hay una disposición constitucional que establece que "La Ciudad asume la responsabilidad indelegable de financiar la educación pública, laica y gratuita en todos los niveles y modalidades a partir de los 45 días de vida, hasta el Nivel Superior, con carácter obligatorio desde el preescolar [...]" (Constitución de la Ciudad de Buenos Aires, Capítulo III, Artículo 24. Convención Constituyente de la Ciudad de Buenos Aires, 1996).

De acuerdo con esta normativa que determina que debe garantizarse la vacante en la educación pública desde los 45 días de edad, la escolarización se ha ido expandiendo hacia extremos impensados, y se ha adelantado a momentos cada vez más próximos al nacimiento. Con base en los datos oficiales de matrícula y en las proyecciones poblacionales disponibles $^{4}$, la tasa de escolarización en 2014 para la edad 5 años en la Caba, es del 92.91\%, estimándose para los niños y las niñas de 4 años de edad una cobertura del 82,99 \%. Para los niños y las niñas de 3 años, actualmente se estima una cobertura del $64.55 \%$, y para el ciclo maternal -de 0 a 2 años-, ésta se limita al $14.71 \%$.

En referencia a los orígenes del Nivel Inicial en América Latina Alberto Martínez-Boom señala que la creación de instituciones oficiales por el Estado "va a generar una demanda que no cesará de crecer" (2004, p. 140), en la Caba parece estar dándose una situación similar. Si bien a medida que se reduce la edad cronológica, la tasa escolar disminuye, la asistencia a la escuela por los más pequeños presenta un crecimiento constante, y el interjuego entre oferta y demanda retroalimentándose puede deducirse de las estadísticas de la Tabla 1:

Tabla 1. Caba. Matrícula salas de 0 a 3 años. Educación común, 2002-20125.

\begin{tabular}{|c|c|c|c|c|c|c|}
\hline & Estatal* & Dif \% & Privado* & Dif \% & \multicolumn{2}{|c|}{$\begin{array}{c}\text { Demanda } \\
\text { Insatisfecha** }\end{array}$} \\
\hline $\mathbf{2 0 0 2}$ & 12.120 & & 15.931 & & 4393 & \\
\hline $\mathbf{2 0 0 3}$ & 12.385 & $2,19 \%$ & 17.582 & $10,36 \%$ & 4970 & $13,13 \%$ \\
\hline $\mathbf{2 0 0 4}$ & 12.316 & $-0,56 \%$ & 18.688 & $6,29 \%$ & 5299 & $6,62 \%$ \\
\hline $\mathbf{2 0 0 5}$ & 12.027 & $-2,35 \%$ & 19.391 & $3,76 \%$ & 6116 & $15,42 \%$ \\
\hline $\mathbf{2 0 0 6}$ & 12.685 & $5,47 \%$ & 20.285 & $4,61 \%$ & 6047 & $-1,13 \%$ \\
\hline $\mathbf{2 0 0 7}$ & 13.467 & $6,16 \%$ & 23.174 & $14,24 \%$ & 6951 & $14,95 \%$ \\
\hline $\mathbf{2 0 0 8}$ & 13.491 & $0,18 \%$ & 23.937 & $3,29 \%$ & 6431 & $-7,48 \%$ \\
\hline $\mathbf{2 0 0 9}$ & 14.275 & $5,81 \%$ & 24.681 & $3,11 \%$ & 4582 & $-28,75 \%$ \\
\hline $\mathbf{2 0 1 0}$ & 14.987 & $4,99 \%$ & 25.063 & $1,55 \%$ & 6091 & $32,93 \%$ \\
\hline $\mathbf{2 0 1 1}$ & 15.897 & $6,07 \%$ & 25.583 & $2,07 \%$ & 5629 & $-7,58 \%$ \\
\hline $\mathbf{2 0 1 2}$ & 17.773 & $11,80 \%$ & 26.657 & $4,20 \%$ & 5518 & $-1,97 \%$ \\
\hline $\mathbf{2 0 0 2}-\mathbf{2 0 1 2}$ & & $46,64 \%$ & & $67,33 \%$ & & \\
\hline
\end{tabular}

* Fuente: Elaboración propia con base en Argentina, Ministerio de Educación. DiNIECE

** ACIJ, Falta de vacantes en el nivel inicial de la Ciudad de Buenos Aires, Buenos Aires, 2013. Hasta 2008 incluye de 0 a 5 años.

\footnotetext{
4 Proyecciones poblacionales provistas por la Gerencia Operativa de Información y Estadística Educativa (Goiee). Ueicee, Ministerio de Educación de Caba.

$5 \quad$ Primer y último año con datos sobre demanda insatisfecha disponibles.
} 
Las cifras de la Tabla 1 reflejan varios aspectos de la expansión cuantitativa de los niños y las niñas escolarizados entre 0 a 3 años de edad en la Caba. En primer lugar, su enorme incremento: en 10 años se han incorporado al sistema educativo más de 16.000 alumnos y alumnas de entre 45 días a 3 años de edad. En segundo lugar, mientras se eleva significativamente la matrícula en las escuelas públicas (46.64\%), para el mismo período se da un crecimiento mayor de la matrícula proveniente del sector de gestión privado (67.33\%). El Nivel Inicial en la Caba no estaría exento de un fenómeno que se repite en el resto de los niveles: la privatización de la matrícula (Narodowski \& Moschetti, 2015), siendo gran cantidad de familias las que deciden asumir el esfuerzo de afrontar una cuota mensual a fin de escolarizar a sus bebés y niños o niñas pequeños. Por último, las cifras de las "listas de espera" denotan una persistente demanda insatisfecha. La tendencia creciente a institucionalizar el cuidado de los más pequeños parece generar una demanda que supera la oferta disponible, independientemente del ritmo de crecimiento de esta última.

Es necesario, además, señalar que la educación inicial en el país se organiza bajo un conjunto sumamente heterogéneo de modos de provisión-configuración que suele reproducirse en el conjunto de la región iberoamericana-, acarreando una gran superposición de responsabilidades e incumbencias de diferentes áreas del Estado, del sector privado y de organizaciones de la sociedad civil (Itzcovich, 2013, Redondo, 2012, Unicef \& Unsam, 2011, entre otros). Mientras que el Nivel Primario se ha expandido sobre la base de un modelo institucional único, el Nivel Inicial sustenta su crecimiento sobre una variedad de dispositivos institucionales (Diker, 2001, Tuñon, 2010).

Específicamente para la Caba, al analizar la cantidad de alumnos y alumnas en Nivel Inicial, es necesario tener en cuenta que gran parte de los bebés y niños y niñas pequeños asisten a modalidades educativas alternativas. La mayor parte de estas ofertas son gestionadas

Rev.latinoam.cienc.soc.niñez juv 15 (1): 45-57, 2017 http://revistalatinoamericanaumanizales.cinde.org.co DOI: $10.11600 / 1692715 x .1510121052016$ desde el ámbito privado $^{6}$, mientras que otro conjunto es ofrecido desde el propio Estado que habilita espacios alternativos al sistema oficial, en su mayor parte, en gestión asociada con Organizaciones de la Sociedad Civil. Son ofertas que podemos agrupar bajo la categoría de no incorporadas a la enseñanza oficial $l^{7}$; es decir que no son reconocidas como instituciones escolares por el Estado, y que en su conjunto, como puede observarse en la Tabla 2, absorben parte importante de la matrícula total del sistema educativo.

Tabla 2. Caba. Nivel Inicial. Educación Común. Matrícula s/ sector de gestión e incorporación a la enseñanza oficial, 2014.

\begin{tabular}{|c|c|c|c|}
\hline & Privado & Estatal & $\%$ \\
\hline $\begin{array}{c}\text { Incorporado a la } \\
\text { enseñanza oficial }\end{array}$ & 68.430 & 52.354 & $81,23 \%$ \\
\hline $\begin{array}{c}\text { No incorporado } \\
\text { a la enseñanza } \\
\text { oficial }\end{array}$ & 17.308 & 10.600 & $18,77 \%$ \\
\hline Total & 85.738 & 62.954 & $100 \%$ \\
\hline
\end{tabular}

Fuente: Elaboración propia con base en Argentina, Ministerio de Educación, DiNIECEy Buenos Aires, Ministerio de Hacienda, DGEyC.

Nota: La categoría No incorporado a la enseñanza oficial incluye Instituciones privadas del Riea, enmarcadas en la Ley $N^{\circ}$ 621; el Programa Primera Infancia del Ministerio de Educación de Caba; los Centros de Primera Infancia, Centros de Desarrollo Infantil y Centros de Acción Familiar del Ministerio de Desarrollo Social.

Considerando la pertenencia o no al sistema de enseñanza oficial y el sector de gestión, la matrícula queda distribuida del siguiente modo (Gráfico 1):

$6 \quad$ Son en su mayoría instituciones que surgieron como guarderías y posteriormente pasaron a regularse por las autoridades educativas según la Ley $\mathrm{N}^{\circ} 621$.

7 La incorporación a la enseñanza oficial en la Argentina se rige por el Decreto No $371 / 64$. "La incorporación es el medio por el cual el Estado reconoce la enseñanza que imparten los institutos privados" (Decreto № 371 de 1964, Capítulo I, Artículo 1). 
Gráfico 1. Caba. Nivel Inicial. Educación Común. Matrícula s/ sector de gestión e incorporación a la enseñanza oficial, 2014.

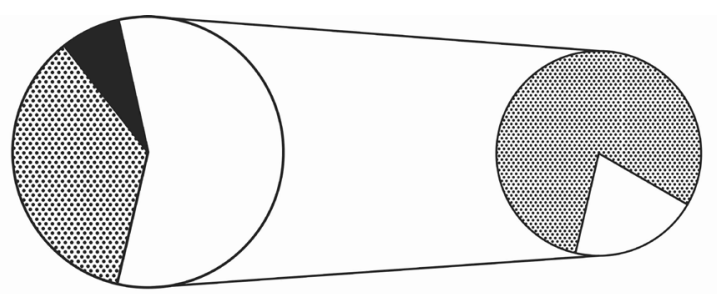

Gestión Privada / 58\%

Gestión Estatal Enseñanza Oficial $/ \mathbf{3 5} \%$

Gestión Estatal Enseñanza No Oficial / $\mathbf{7 \%}$

Fuente: Elaboración propia en base a Argentina, Ministerio de Educación, DiNIECE y Buenos Aires, Ministerio de Hacienda, DGEyC.

El Gráfico 1 demuestra que un 19\% de los alumnos y alumnas asiste a instituciones que son reconocidas por las autoridades como no oficiales. Además, el sector privado cobra un protagonismo impactante absorbiendo un 58\% del total de la matrícula.

Por otro lado, sibien el sector no incorporado a la enseñanza oficial gestionado por parte del Estado representa al momento tan solo un 7\% del total, no puede dejar de mencionarse que el crecimiento de estas modalidades en los últimos años ha sido sumamente relevante. En la Caba, a fin de dar respuesta al déficit de cobertura, el Gobierno ha adoptado una política de optimización de los recursos limitados disponibles mediante la apertura de estas modalidades alternativas ${ }^{8}$, dirigidas con exclusividad a poblaciones con un alto índice de necesidades básicas insatisfechas. Esta política costo-efectiva ha generado controversias desde diferentes actores sociales, poniendo en discusión la calidad de los servicios que se ofrecen, ya que responden a lógicas de menores exigencias en cuanto a la infraestructura,

$8 \quad$ Una de las principales modalidades de gestión asociadas con ONGs son los Centros de Primera Infancia, dependientes del Ministerio del Desarrollo Social. Fueron creados en el año 2009 mediante el Decreto $\mathrm{N}^{\circ}$ 306. La matrícula a mediados del 2014 era de 6400 niños y niñas (www.buenosairesciudad.gob). Este dato, en relación con los 3.000 niños y niñas atendidos para el 2011, estaría indicando un crecimiento del $213 \%$ en tres años. a los niveles de profesionalización del personal a cargo de los niños y las niñas, y a las supervisiones pedagógicas (Faur, 2010, Redondo, 2012, Repetto, Langou \& Aulicino, 2012, Sverdlick, 2007, entre otros).

En síntesis, la obligación que compele al Estado de la Ciudad de Buenos Aires a garantizar las vacantes desde los 45 días de vida, el significativo crecimiento de la escolarización temprana acompañado de la privatización de la matrícula, las diversas formas de oferta educativa en que se da respuesta a una demanda siempre insatisfecha, ha vuelto a la escolarización de la población en sus primeros años de vida en la Caba objeto de debates políticos y reclamos sociales. A su vez, la constituye como un caso paradigmático a los fines de analizar sentidos subyacentes en esta tendencia a escolarizar bebés y niños y niñas pequeños.

\section{Sentidos explícitos: al jardín se va a aprender}

No es necesario retroceder muchos años para dar con una época en que la familia era considerada el espacio privado privilegiado para la educación de los niños y las niñas más pequeños, y se esperaba que fueran los padres y madres -especialmente éstas- quienes asumieran la responsabilidad por las tareas de crianza. Sin embargo, como señala Castro, Vander-Veer, Burgos-Troncoso, Meneses-Pizarro, Pumarino-Cuevas \& Tello-Viorklumds (2013), las formas en que se cría a los niños y las niñas cambian según la cultura, los modelos mentales y los nuevos inventos tecnológicos.

La institucionalización de los bebés y los niños y las niñas pequeños surgió a partir de la necesidad de sostener la crianza por un medio alternativo al del grupo familiar de pertenencia (Argentina, Ministerio de Educación y Justicia \& OEA, 1986, López, 2004, Unicef \& Unsam, 2011). Pero el ingreso de la mujer a la fuerza laboral, responde al interrogante del crecimiento sostenido y pronunciado de la demanda, tan solo en parte. Por ejemplo, la extensión de la licencia con goce de sueldo por maternidad, bien podría ser uno de los focos de los reclamos por los derechos de la infancia: extender el salario durante meses (o años) mientras perdure 
la más exigente función materna. Sin embargo, lo que se impone en la agenda política es el reclamo de vacantes en instituciones educativas (Snaider, 2014).

En este sentido, María Emilia López (2004) señala que hay un desplazamiento con respecto al origen de los jardines maternales: "No se inventaron porque algún equipo de especialistas descubrió que con escolarización más temprana habría mejores aprendizajes, garantías de mayores índices de alfabetización, ciudadanos en mejores condiciones de llegar al mundo laboral" (López, 2004, p. 1).

En línea con este desplazamiento señalado, las autoridades atribuyen la demanda creciente a la existencia de un cambio cultural que lleva a que "cada vez más, los padres elijan dejar a sus hijos en los jardines maternales o escuelas infantiles antes que con un familiar o miembro de su comunidad inmediata" (Repetto et al., 2012, p. 48). Cambios culturales que se dan en relación con la familia, la maternidad, las pautas de crianza, la concepción de la niñez y su desarrollo, y la función que cumplen las instituciones de educación inicial.

Hacia finales del Siglo XX se fue avanzando en la construcción de un paradigma diferente: se fue dejando atrás un modelo tutelar en el que el niño o niña era considerado tan sólo un "menor" y un sujeto pasivo, y se modificó la representación de la infancia reivindicando su carácter como sujeto de derechos $^{9}$ (López, Rodríguez \& Tomassino, 2012, Malajovich, 2010). En este contexto, las ciencias "psi", como la psicología infantil y la pedagogía, contribuyeron a modificar la concepción del niño o niña centrando la mirada en sus necesidades, y sustentaron la definición de la identidad pedagógica del Nivel Inicial, intentando el reconocimiento de los potenciales de aprendizaje en los primeros años de vida y las consecuencias de no ofrecer la estimulación adecuada en esta etapa crítica de la vida (Diker, 2001, Malajovich, 2010).

La temprana institucionalización ya no puede concebirse tan sólo como relevo de los sujetos adultos responsables de la crianza

$9 \quad$ Existe consenso con respecto al hecho de que la Convención de los Derechos del Niño en el año 1989 es uno de los hitos en ese sentido. durante su jornada laboral, "sino como reconocimiento del sentido que tiene para el desarrollo infantil la participación de los niños y las niñas en situaciones educativas valiosas y variadas a cargo de personal especializado" (Terigi, 2012, p. 126). Los debates acerca de la especificidad pedagógica del nivel instalarán definitivamente la idea de que la educación inicial cumple una función en relación con el desarrollo evolutivo infantil, y en esta línea se fue avanzando "de la educación inicial para pocos, a la educación para todos" (López et al., 2012, p. 5).

Es en este contexto que desde fines de los años sesenta del siglo $\mathrm{XX}$, las prácticas que tradicionalmente eran circunscriptas a la acción familiar-maternal se fueron desplazando progresivamente a favor de su incorporación en el sistema educativo (Martínez-Boom, 2004, Narodowski, 2013).

Sin embargo, este adelantamiento del ingreso de los niños y las niñas a la escuela es paradojal, en el sentido de que se desarrolla en un contexto de discursos extendidos sobre la crisis de la institución escolar (Terigi, 2012). Al mismo tiempo en que el formato escolar se encuentra sumamente cuestionado, también la legitimidad estatal que supo sustentar la creación y expansión de los sistemas educativos nacionales se halla en crisis. De todas maneras se observa que la escolarización se adelanta, y conlleva la extensión del principio de infancia como razón de Estado ${ }^{10}$ a edades cada vez más tempranas (Narodowski, 2013). Esto refuerza, a nuestro entender, la hipótesis de que no sólo no se ha encontrado aún una idea superadora para pensar lo escolar, sino que el fenómeno en cuestión requiere ser comprendido en un contexto de cambios culturales más profundos. Es dable preguntarse cuáles son los cambios culturales y demográficos implícitos en los argumentos de más vacantes disponibles; adoptar un abordaje situado y contextual, una perspectiva en la que adquieran centralidad los entramados relacionales y las especificidades del contexto (Ospina \& Aguilar-Forero, 2015), para comprender por qué aquellos tiempos en que se esperaba que las mujeres de la

10 Para mayor profundidad en este concepto, véase Narodowski (1999). 
familia educaran a sus bebés, son percibidos por las nuevas generaciones como tiempos exageradamente lejanos y completamente ajenos.

\section{Sentidos implícitos: adiós al sacrificio paterno}

Este nuevo escenario de escolarización de bebés y niños a edades cada vez más tempranas supone que padres y madres ya no se encargan directamente de sus hijos e hijas, sino que lo hacen por intermedio de una escuela y de unos maestros y maestras. ¿Es por una necesidad laboral? ¿Es porque actualmente se entiende que el jardín cumple una función pedagógica esencial? Es probable. Pero aun así, es necesario entender el nuevo escenario en el contexto de una cultura que ya no legitima el sacrificio paterno por el bienestar de los hijos e hijas.

Aún en el caso de padres y madres de muy bajos recursos económicos, la vieja cultura posfigurativa -para la cual el valor supremo es la experiencia y la adultez y la vejez deben ser honradas y admiradas- (Mead, 1970, Narodowski, 2011), designaba con la palabra "sacrificio" aquella característica de la actividad paterna por medio de la cual los adultos resignan bienestar propio a favor del bienestar, y en última instancia, de la felicidad de sus hijas y sus hijos. Estos se encuentran, se supone, en una posición de vulnerabilidad y de necesidad de cuidado tal, que la persona adulta a cargo no solamente requiere constituirse en un ejemplo legítimo sino también "darlo todo" y "darse a sí misma" por el bien de los hijos y las hijas.

El desiderátum de la sociedad moderna respecto de la protección a de los menores lo configuraron los adultos (padres, madres, docentes) que lo daban todo -en un sentido literal- por sus menores a cargo (hijos e hijas, alumnos y alumnas); que se brindaban en cuerpo y alma por ellos; que estaban dispuestos a ejercer sacrificios absolutos, pagados a veces con su salud o con su vida misma, por el bienestar y la felicidad de sus hijas e hijos (Narodowski, 2011).

Como hemos analizado en otro trabajo (Narodowski, 2016), el Holocausto/Shoá, como toda gran tragedia de la modernidad, ha brindado testimonios múltiples respecto del sacrificio de padres, madres y docentes en pos del bienestar de hijos e hijas, y alumnos y alumnas. Entre ellos, es ampliamente conocida la epopeya de Janusz Korczak quien, a pesar de haber salvado su propia vida, la opción por los niños que tenía a cargo ("mis niños", dirá la leyenda que los llamaba el Maestro Korczak) lo llevó a acompañarlos a la cámara de gas y, por lo tanto, a morir junto a ellos, garantizando, en ese periplo, el mayor cuidado y protección posible (Naranjo, 2001).

En esta vieja cultura posfigurativa a los niños y las niñas se les protege. Al fin de cuentas, Janusz Korczak no hizo otra cosa que ser consecuente con su propia idea de amor y cuidado de los niños, aún en el peor de todos los escenarios posibles: en sus libros y escritos este Maestro nos había mostrado cuál era la forma correcta y verdaderamente humana de amar a los niños y las niñas ${ }^{11} \mathrm{y}$, consecuente con sus propios postulados, tuvo la valentía de llevar sus ideas hasta sus últimas consecuencias trágicas.

Ya en el plano de la ficción, otro ejemplo que conforma un panegírico de la protección de los niños y las niñas para nuestra cultura moderna lo constituye la película ganadora de tres premios de la Academia La vida es Bella, de Roberto Benigni ${ }^{12}$. La obra tiene la enorme virtud de dramatizar la experiencia en un campo de concentración nazi, en el que un prisionero judío hace lo imposible para que su hijo, prisionero junto a él, no solamente sufra menos que los adultos, sino que, en la medida de lo posible, ni siquiera perciba la naturaleza de los horrores a los que está asistiendo y de los que es víctima. También en este caso la protección de la pureza infantil es absoluta, como igualmente lo es el sacrificio de su padre, quien relega las pequeñas porciones de bienestar personal a las que podría acceder en ese infierno, a favor del bienestar de su hijo y, recíprocamente, opta por mayores pesares en su detención en la medida en que eso le pueda servir para ahorrarle mayores pesares al niño.

11 Puede verse, por ejemplo, Janus Korszac (1985).

12 Roberto Benigni (Director), La vita è bella [Película], Miramax International, 1997.

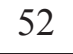

Rev.latinoam.cienc.soc.niñez juv 15 (1): 45-57, 2017 http://revistalatinoamericanaumanizales.cinde.org.co DOI: $10.11600 / 1692715 x .1510121052016$ 
En rigor, la película La Vida es bella parece terminar mostrando una suerte de posición conscientemente autista, en la que al niño se le pretende ahorrar el más elemental principio de realidad con tal de salvaguardar la entereza de la puerilidad infantil: para los niños y las niñas, la vida siempre debe ser bella.

Es que es en el sacrificio en donde estaba sólidamente basado el amor a los hijos e hijas. Por eso, la queja frente al sacrificio no era procedente ya que en el sacrificio adulto era donde padres, madres y docentes hallaban su identidad como tales. Las letras del Tango Argentino son bien elocuentes de cómo la cultura popular recogía la experiencia de ser padres o madres, y de ser hijo o hija, reconociendo el sacrificio como uno de sus principales pilares. Entre muchas otras puede escucharse:

Sentí desde niño confianza en la vida

estando a tu lado ¿qué pude temer?

yo vi el sacrificio

yo vi tus heridas

luchando con todo

para no caer ${ }^{13}$.

Este viejo sacrificio adulto no implicaba una posición de mera provisión o una entrega ilimitada de bienes y símbolos. Al contrario, el sacrificio adulto en pos de los niños y las niñas era enteramente racional, y muchas veces implicaba la necesidad de negar lo que los niños y las niñas solicitaban, y hacerlo únicamente por su bien. Negación de la provisión de bienes, de actividades, de símbolos, incluso de cariño -aun poseyendo lo demandado- era el máximo sacrificio posible. Sacrificio por partida doble: para garantizar el bienestar y el beneficio presente o futuro de hijos e hijas, y alumnos y alumnas, se brindaba todo lo que las personas menores necesitaban -incluso lo que no se tenía-y, con dolor, se les negaba a los niños y las niñas aquello que a veces se poseía, si es que se consideraba perjudicial para ellos y ellas.

La respuesta esperada, efectivamente constituida y que se construye por la infancia respecto del sacrificio paterno ha sido, a la vez, el respeto y el agradecimiento: el sacrificio por el otro-niño ha formado parte de la constitución

13 Padre mío, letra y música de Ricardo Cardé. Disponible en http://www.tangoletras.com.ar/letra-de-padre-mio-versioncompleta-6277 [Consultado 27/05/2016].

Rev.latinoam.cienc.soc.niñez juv 15 (1): 45-57, 2017

http://revistalatinoamericanaumanizales.cinde.org.co

DOI: $10.11600 / 1692715 \times .1510121052016$ misma de la identidad adulta de nuestra cultura posfigurativa, y lo único que pretende a cambio es apenas el reconocimiento simbólico de su existencia. El sacrificio paterno o materno no demandaba ni requería compensación material, aunque sí es esperable por un "buen hijo" o de un "buen alumno" el reconocer lo actuado por el otro-adulto. Reconocimiento que, en muchas ocasiones, no era posible en el momento en el que se atravesaba el lugar filial o pedagógico, pero que de alguna manera debía materializarse -y así acontecía- como parte de la llegada de ese individuo menor al mundo adulto.

Es cierto que el sacrificio en función de lo infantil no era representado unívocamente en nuestra vieja modernidad posfigurativa. Muchas investigaciones -especialmente las de carácter etnográfico- que intentaron captar la dinámica íntima de los procesos de apropiación de la cultura escolar por parte de los sectores sociales más empobrecidos de la población, han encontrado que el sacrificio no era naturalmente aceptado y las resistencias eran múltiples.

Pero este tipo de sacrificio no es equivalente al sacrificio adulto en función de sus descendientes y del alumnado: mientras uno es entrega, compromiso y postergación ilimitada del deseo, el sacrificio de los más pobres en el sistema educativo no es otra cosa que desacomodamiento e impaciencia frente a una situación escolar tan extrañamente diferente a sus experiencias culturales y de clase social. En este caso, el diferimiento no es sine die, como el de los individuos adultos de la modernidad -que a lo sumo sentían la satisfacción del deber cumplido-, sino que tiene un plazo fijo más cercano o más lejano, dependiendo de la situación.

Por supuesto, la sociología ha producido diversos estudios que muestran con evidencia confiable que este sacrificio adulto tiene diferentes formas de realización en contextos socioculturales diversos: el estudio ya clásico de Sue Middleton, Ashworth y Braithwaite (1997) muestra, para el caso inglés, que en términos relativos el sacrificio de los padres y las madres respecto de sus hijos y sus hijas es inversamente proporcional a su nivel socioeconómico; en otras palabras, cuanto más pobre es un hogar, una proporción mayor de sus 
recursos se destinará al cuidado de los hijos y las hijas. La investigación encontró, incluso, padres y madres de sectores socioeconómicos muy bajos, que en los hechos son más pobres que los propios hijos e hijas bajo su cuidado.

El mandato cultural posfigurativo era, pues, de cumplimento inexorable para quienes ocuparan el lugar de personas adultas: hacia las hijas y los hijos, el sacrificio. Por supuesto, en el mundo adulto existía una división de roles y funciones al respecto, que fueron variando en función de épocas y de espacios territoriales; las madres cumplían funciones diversas respecto de los padres o de los otros miembros de la familia, y los maestros y maestras, a su vez, se diferenciaban por su actuación de todo el grupo familiar. Pero el conjunto adulto reproducía pautas posfigurativas de crianza, y el sacrificio era una parte ineludible de ellas. Incluso aquellos sujetos adultos que no tenían hijas o hijos eran sindicados como responsables por los hijos e hijas de los otros. No hablamos ya de los sacerdotes católicos (romanos) que en virtud del celibato no tienen hijos ni hijas y que, paradojalmente, y siguiendo la línea explicativa aquí trazada, eran (y son) llamados padres. Incluso aquellos que por vicisitudes de la vida social no alcanzaron el grado de paternidad que era visto como normal para la vida adulta -el tener hijos no era una elección-: a ellos también se les requería, de hecho, un compromiso. Al menos con su obligación de no dejar de "dar el ejemplo".

Al contrario, quienes no cumplieren con este mandato eran percibidos bajo una conducta caracterizada como "inapropiada" desde el punto de vista moral. Incluso las sociedades podían disponer de recursos legales y coactivos que podrían llegar a acarrear severos trastornos hacia padres y madres que no protegieran a sus hijos e hijas, implicando desde la pérdida de su tutela hasta penas de multa o prisión, cuando se consideraba que supeditar el beneficio propio en detrimento de la felicidad de los hijos e hijas ponía en riesgo la salud física o psíquica de las personas menores.

Pero las cosas han cambiado y el autogobierno y la responsabilidad por los propios actos ya no son un valor que merezca ser destacado, y mucho menos lo será el sacrificio para que otros alcancen ese devaluado logro. Nuestra cultura ahora es prefigurativa en los términos de Mead (1970), y apenas considera el sacrificio por los hijos e hijas y por los alumnos y alumnas, una acción que a veces es menester ejercer inexorablemente porque en ciertos márgenes etarios o actitudinales aún no es posible confiar en los niños, niñas o adolescentes una práctica susceptible de reconocimiento (Narodowski, 2016). Ya nadie se vanagloria públicamente ni parece sentirse orgulloso íntimamente por las privaciones o las postergaciones a las que tuvo que acometer por el hecho de criar a una persona menor.

¿Qué queda pues, del sacrificio por los hijos y las hijas, por los más pequeños, como virtud propia del viejo mundo adulto? Cada vez menos, en la crianza familiar de los bebés, que tiende a ser "tercerizada" en instituciones escolares especializadas en recién nacidos. Así, el sacrificio sólo se ejerce frente a situaciones extremadamente delicadas, por lo que hasta la misma palabra "sacrificio" necesita de no pocos aditamentos para mantener su vigencia. Para la vida cotidiana, lo ideal es contar con recursos (humanos, tecnológicos, financieros, institucionales) que sustituyan el sacrificio adulto por la comodidad de las soluciones de la crianza pret à porter: Las escuelas maternales cumplen buena parte de esta expectativa, sobre todo en sectores medios y altos de la sociedad no acuciados por el trabajo paterno/materno y donde la asistencia del bebé al jardín es una opción no compulsiva. Futuras investigaciones deberían dar cuenta pormenorizadamente de esta cuestión.

¿Es este cambio en las pautas de crianza y en la escolarización de bebés un giro que tiende a la estatalización de los cuerpos? (Foucault, 1985). Es difícil sostener esta hipótesis; más bien tendemos a conjeturar que una sociedad post sacrificial -en el sentido de Lipovetsky (1992) - parece sostener cada vez menos ligazones entre viejas y nuevas generaciones que tiendan a que aquéllos se sacrifiquen $-\mathrm{O}$ sacrifiquen sus pretensiones- en función del cuidado y la protección de éstos. El Estado, por su parte, parece construir una respuesta blanda, de satisfacción del cliente, para sostener esta elusión respecto de los roles prefigurativos en la crianza de los bebés. 


\section{Lista de referencias}

Argentina, Ministerio de Educación y Justicia \& OEA (1986). Situación de los Jardines Maternales en CapitalFederal, 1984-1985. Recuperado de: http://www.bnm.me.gov. ar/giga1/documentos/EL003336.pdf

Brailovsky, D. (2006). Revoluciones silenciosas. El jardín de infantes y el homeschooling como tentativas de reinvención de lo escolar. En M. Narodowski \& D. Brailovsky (comps.) Dolor de Escuela, (pp. 131-152). Buenos Aires: Prometeo Libros.

Brailovsky, D., Herrera, S., Salinas, S. \& Santa Cruz, E. (2002). Didáctica de la crisis. Buenos Aires: Novedades Educativas.

Burgos, N. \& Silva, M. (2013). Los maestros del nivel inicial, entre la cualificación y la proletarización. Reflexiones sobre debates que continúan vigentes. RELAdEI, Revista Latinoamericana de Educación Infantil, 2, pp. 27-38.

Caputo, M. \& Gamallo, G. (2010). La calidad del jardín maternal y su influencia en el desarrollo cognitivo de los niños y las niñas. Revista Latinoamericana de Ciencias Sociales, Niñez y Juventud, 8 (2), 849-860. Recuperado de: URL http:// revistaumanizales.cinde.org.co/index. php/Revista-Latinoamericana/article/ view/75/33

Castro, P. J., Van-der-Veer, R., BurgosTroncoso, G., Meneses-Pizarro, L., Pumarino-Cuevas, N. \& TelloViorklumds, C. (2013). Teorías subjetivas en libros latinoamericanos de crianza, acerca de la educación emocional. Revista Latinoamericana de Ciencias Sociales, Niñez y Juventud, 11 (2), pp. 703-718. Doi :10.11600/1692715x.11217060213.

Congreso Nacional de Argentina (2006). Ley de Educación Nacional 26.206, Artículo 18. Buenos Aires: Congreso Nacional de Argentina.

Convención Constituyente de la Ciudad de Buenos Aires (1996). Constitución de la Ciudad Autónoma de Buenos Aires. Buenos Aires: Convención Constituyente de la Ciudad de Buenos.
Diker, G. (2001). Organización y perspectiva de la Educación Inicial en Iberoamérica, principales tendencias. Madrid: OEI. Recuperado de:

http://www.oei.es/inicial/articulos/

Faur, E. (2010). Lógicas en tensión. Desencuentros entre oferta y demanda de servicios de cuidado infantil en Buenos Aires. Revista de Ciencias Sociales, 27, pp. 68-81.

Foucault, M. (1985). Vigilar y Castigar. Nacimiento de la prisión. México, D. F.: Siglo XXI.

Itzcovich, G. (2013). La expansión educativa en el nivel inicial durante la última década, Cuaderno $n^{\circ}$ 16, Sistema de Información de Tendencias Educativas en América Latina. Recuperado de: http://www.siteal.iipe-oei.org/sites/ default/files/siteal_cuaderno_16_nivel_ inicial.pdf

Korszack, J. (1985). Cómo amar a los niños. México, D. F.: Trillas.

Lipovetsky, G. (1992). Le crépuscule du devoir. L'étique indolore des nouveaux temps démocratiques. París: Gallimard.

López, M. E. (2004). Los bebés en el jardín. Revista Punto de Partida, (2). Recuperado de:

h t t p : / / w w w. colegioimi.net / uploads/2/3/2/3/23231948/los_bebes_ maternal.pdf

López, N., Rodríguez, J. \& Tomassino, M. (coords.) (2012). La Situación de la Primera Infancia en Argentina. A dos décadas de la ratificación de la Convención de los Derechos del Niño. Córdoba: Fundación Arcor. Recuperado de:

http://www.sipi.siteal.org/sites/default/ files/sipi_publicacion/informe_arcor_0.pdf Malajovich, A. (2010). Deudas educativas con la primera infancia. Voces en el Fénix, 3, pp. 34-37.

Martínez-Boom, A. (2004). De la escuela expansiva a la escuela competitiva: Dos modos de modernización educativa en América Latina. Bogotá, D. C.: Anthropos.

Mayol-Lasalle, M. (2002). Legislación del cuidado y la educación temprana: Una deuda con la primera infancia. En L. Pitluk 
(comp.) La Educación Maternal III. Un Desafio a Favor de la Primera Infancia, (pp. 39-52). Buenos Aires: Novedades Educativas.

Mead, M. (1970). Cultura y compromiso. El mensaje de la nueva generación. Barcelona: Gedisa.

Middleton, S., Ashworth, K. \& Braithwaite, I. (1997). Spending on Children, Childhood Poverty and Parental Sacrifice. York: Joseph Rowntree Foundation.

Naranjo, R. (2001). Janus Korszack, maestro de la humanidad. Buenos Aires: Novedades Educativas.

Narodowski, M. (1999). Después de clase. Desencantos y desafios de la escuela actual. Buenos Aires: Novedades Educativas.

Narodowski, M. (2011). No es fácil ser adulto. Revista de Educación y Pedagogía, (Medellín), 23 (60), pp. 101-114.

Narodowski, M. (2013). Políticas públicas e infancias: Deseos y límites a la igualdad en educación. Revista Brasileira de Educação, 18 (54), pp. 551-570. Doi: 10.1590/S1413-24782013000300003.

Narodowski, M. (2016). Un Mundo sin adultos. Familia, escuela y medios frente a la desaparición de la autoridad de los mayores. Buenos Aires: Debate.

Narodowski, M. \& Moschetti, M. (2015). The growth of private education in Argentina: Evidence and explanations. Compare: A Journal of Comparative and International Education, 45 (1). Doi: 10.1080/03057925.2013.829348.

OEI, Cepal \& Secretaría General Iberoamericana (2010). 2021, Metas Educativas. La educación quequeremos para lageneración de los bicentenarios. Documento Final. Madrid: OEI. Recuperado de: http://www. oei.es/metas2021/libro.htm

Ospina, H. F. \& Aguilar-Forero, N. (2015). Editorial. Revista Latinoamericana de Ciencias Sociales, Niñez y Juventud, 13 (2), pp. 535-544. Recuperado de:

http://revistaumanizales.cinde.org.co/ index.php/Revista-Latinoamericana/ article/view/2034/631

Pitluk, L. (2002). El jardín maternal: Una institución educativa. En L. Pitluk (comp.)
La Educación Maternal III. Un Desafío a Favor de la Primera Infancia, (pp. 6-21). Buenos Aires: Novedades Educativas.

Ponce, R.(2006).Losdebates delaeducaciónen la Argentina. Persistencias, transformaciones y resignificaciones a lo largo de la historia. En A. Malajovich (comp.) Experiencias y reflexiones sobre la educación inicial: Una mirada latinoamericana, (pp. 19-102). Buenos Aires: Siglo XXI.

Redondo, P. (2012). Políticas en debate: La atención educativa de la primera infancia en la Argentina. Propuesta Educativa, 37, pp. 6-16.

Repetto, F., Langou, G. D. \& Aulicino, C. (2012). Cuidado Infantil en la Ciudad Autónoma de Buenos Aires ¿La disyuntiva entre pañales y pedagogía? Buenos Aires: Cippec.

Snaider, C. (2014). El nivel inicial en la Ciudad de Buenos Aires. Lógicas que ordenan ofertas crecientes y diversas. Tesis para optar el título de Magister en Administración de la Educación, Escuela de Gobierno, Universidad Torcuato Di Tella, Buenos Aires, Argentina.

Sverdlick, I. (coord.) (2007). La situación de la primera infancia en la provincia de Buenos Aires. La acción de las organizaciones $y$ movimientos sociales. Buenos Aires: Unipe. Recuperado de: http://unipe.edu. ar/wp-content/uploads/2011/11/IngridSverdlick.pdf

Terigi, F. (2012). La escolarización como política hacia la primera infancia. En E. Duro (ed.) Crecer Juntos para la Primera Infancia. Encuentro Regional de Políticas Integrales 2011, (pp. 125-129). Buenos Aires: Unicef.

Tuñón, I. (2010). Determinantes de las oportunidades de crianza y socialización en la niñez y en la adolescencia. Revista Latinoamericana de Ciencias Sociales, Niñez y Juventud, 8 (2), pp. 903-920. Recuperado de:

http://revistaumanizales.cinde.org.co/ index.php/Revista-Latinoamericana/ article/view/79/37

Unicef \& Unsam (2011). Servicio de Atención a Niños y Niñas de 45 días a 36 meses. 
Recuperado de: http://www.unicef.org/ argentina/spanish/Serv_Aten_Ninos_web. pdf

Unión Europea (2013). Recomendación de la Comisión Europea. Invertir en la infancia: Romperel ciclodelas desventajas. Bruselas: Diario Oficial de la Unión Europea. Recuperado de: http://www.lamoncloa. gob.es/espana/eh15/politicasocial/ Documents/Recomendacion_de_la Comision_Europea.pdf 